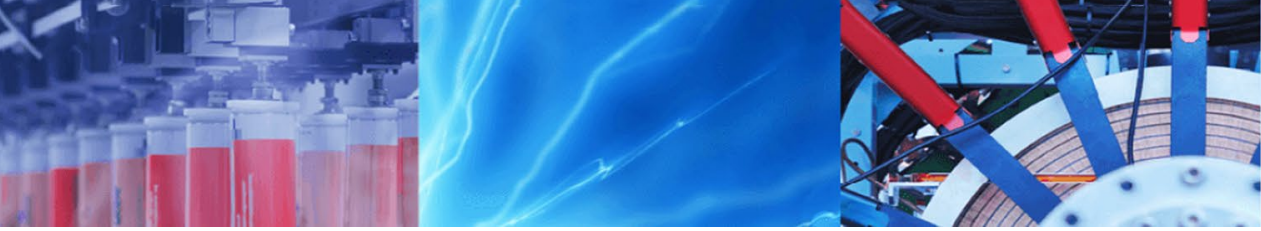

Research Article

\title{
Experimental investigation on the influence of a "soft layer" on the structural performance of a smart composite structure
}

\author{
X. Chen ${ }^{1,2} \cdot$ Y. Meyer $^{1,3}$ (I) R. Lachat ${ }^{4} \cdot$ M. Ouisse ${ }^{2}$
}

() Springer Nature Switzerland AG 2019

\begin{abstract}
The composite structures embedding piezoelectric implants are developed due to their abilities of modifying mechanical properties according to the environment, of keeping their integrity, of interacting with human beings or with other structures. One way to functionalize a mechanical device consists in embedding the transducers inside the final composite structure via a "soft" layer. This layer consists of two plies sandwiching the transducers, impregnated with a resin compatible with the one of the final composite structures. The test structures are laminates made of a glass-fiber reinforced plastic with a polyester resin. In this paper, we propose to experimentally investigate the influence of the throughthe-thickness position of the "soft layer" on specific parameters of design such as eigenfrequencies, modal amplitude, damping ratio and Lamb wave propagation properties. Results show that the "soft" layer behavior can not be neglicted to predict the behavior of the final product in particular for the eigenfrequencies and the modal amplitudes. However, the "soft layer" has no impact on the damping ratio and the Time-of-Flight of a wave train.
\end{abstract}

Keywords Experimental investigation · Smart composite · Soft layer · Laminates · Piezoelectric transducer

\section{Introduction}

Currently, in many industrial fields such as transportation, a research effort is conducted to reduce the structural weight $[5,41]$. Composite materials turn to be one of the most interesting solutions because their mass density is low and their stiffness is high. A composite material can be defined as a combination of two or more materials, in general strong fibers embedded in a weaker matrix, that results in better properties than those of the individual components used alone $[4,15]$. However, these materials have also some shortcomings such as weakness to delamination, low-velocity impact resistance or low damping ratio $[16,22]$. It is then of first importance to monitor the structural health of these composite structures. This can be done by designing and manufacturing composite structures with a distributed set of integrated transducers $[2,14]$. These smart composite structures have the abilities of modifying mechanical properties according to the environment (e.g. active vibration control [10]), of keeping their integrity (e.g. structural health monitoring [18]), of interacting with human beings (e.g. Human-Machine Interaction [3]) or with other structures (e.g. mechatronic [46]). This paper is focused on composite structures with embedded bulk piezoelectric transducers [11].

One key point of this technology is the way of embedding the smart materials during the manufacturing process. The easiest method consists in directly placing the transducers between two plies, but resin pockets usually appear at the transducer boundaries, which can create structural weaknesses [32]. Moreover, the transducer location is not accurately guaranteed because the

$\triangle$ Y. Meyer, yann.meyer@gmail.com | ${ }^{1}$ UTBM, Univ. Bourgogne Franche-Comté, 90010 Belfort, France. ${ }^{2}$ FEMTO-ST Institute, CNRS/UFC/ENSMM/UTBM, Univ. Bourgogne Franche-Comté, Besançon, France. ${ }^{3}$ CNRS, FRE 2012 Roberval, Centre de recherche Royallieu, Sorbonne Universités, Université de Technologie de Compiègne, CS 60 319, Compiègne cedex, France. ${ }^{4}$ ICB UMR 6303, CNRS, UTBM, Université de Bourgogne Franche-Comté, 90010 Belfort, France. 
piezoelectric elements can move during the compaction and resin spread. Another method is to use a ply with cutout, corresponding to the exact geometry of the transducer, but some discontinuities are created in the fiber layer [17]. An analytical study performed by Chow and Graves has proved that the insertion of transducers can affect the integrity of smart structures [8]. The results show that the magnitude of inter-laminar stresses in a graphite/ epoxy laminate increases by five times, due to the presence of embedded inert rectangular implant. Hansen and Vizzini [20] have performed static tension and tensiontension fatigue tests on carbon/epoxy composites with inserted glass slices. Their results show that embedding techniques have significant influence on the static and fatigue strengths of the composites. Particularly, compared with interlacing technique, cut-out method can significantly degrade the fatigue life of embedded composites. Moreover, for complex structures, the geometry of the cut-out and the accurate positioning of the transducers can be difficult to obtain. Specific manufacturing methods have been developed to place the transducers system at the heart of the composite material. Stanford Multi-Actuator-Receiver Transduction Layer ("SMART Layer") has been developed by Lin and Chang [27], which is used to integrate a network of distributed piezoceramic transducers into the heart of graphite/epoxy composite laminates in their manufacturing process. For this, a semifinished product based on a polyimide encapsulation for the transducers, is created during a supplementary manufacturing step. They have demonstrated that the embedded transducers can be used without degrading the structural integrity of the host composite structures [36]. But, this solution needs to pay a particular attention to the ratio between the semi-finished product surface and the overall product surface because the encapsulation material and the matrix do not have the same chemical nature. If this ratio is too high, delamination problems could occur. In this work, a glass-fiber reinforced plastic (GFRP) composite with two plies sandwiching the transducers and impregnated with a resin compatible with the one of the final composite structure has been used to create a "soft layer" as a semi-finished product $[6,24,29,31,32,37]$.

From an industrial point of view, it is essential to understand the influence of the "soft layer" on the operating performance of the smart composite structures in order to manage it during the design stages of the product lifecycle and to integrate it in the technical requirements [30]. The effect of integrated transducers on the mechanical behaviour of composite structures has been extensively studied and reported from a numerical point of view [23, $34,38,39]$. From the authors' knowledge, much less attention has been devoted to experimentally evaluate it [21]. This article is focused on the experimental investigation of the impact of the through-the-thickness location of the "soft layer" on the final performance of the smart composite structure. Furthermore, the results obtained can constitute an experimental benchmark data set that will be useful for validation of computational codes or model developments.

The paper is organized as follows. The description of the samples tested and the composite manufacturing process are introduced in Sect. 2. Section 3 describes the experimental technique used for the samples characterization and the data correction used to compare the experimental data. In Sect. 4, the results are presented, compared and discussed. Finally, concluding remarks are given.

\section{Samples description and manufacturing process}

In order to study the influence of the "soft layer" on the structural performance of the smart composite structures, a set of smart composite beams are manufactured. This section describes the geometry of the beams and their manufacturing process.

The worldwide production of composite structures reached around 10 millions tonnes in 2016. Glass fibers are still by far the most commonly used reinforcing material in fiber reinforced plastics and composites (More than $90 \%$ of all composites) $[1,45]$. $70 \%$ of composite structures are made of thermoset polymer matrix, in particular unsaturated polyester resins $[1,45]$. This is the reason why this work is focused on laminates made of a glass-fiber reinforced plastic (GFRP) with a polyester resin.

\subsection{Samples description}

As presented in Fig. 1, the test beams are $50 \pm 1 \mathrm{~mm}$ wide, $715 \pm 1 \mathrm{~mm}$ long and $2.5 \pm 0.05 \mathrm{~mm}$ thick. The composite material consists of Glass Fiber Reinforced Polymer (GFRP) laminate. It was prepared from 6 plies of chopped strand mat. The matrix is a thermosetting plastic (polyester resin) Each ply is $0.33 \mathrm{~mm}$ thick. The "soft layer" is $0.5 \mathrm{~mm}$ thick because of the transducers thickness $(135 \mu \mathrm{m})$ and mainly made of polyester resin because the two fiber plies used to sandwich the transducers and the wires are very light (surface mass of $30 \mathrm{~g} \mathrm{~m}^{-2}$ ). The piezoelectric transducers have been characterized and all the material parameters are given in [28]. Due to the low standard deviations for the different material parameters, the transducers from different production batchs can be considered identical. For each beam, three piezoelectric elements are embedded inside the beams at the same depth. This latter parameter is modified for each beam reference. The location of the "'soft layer"' for each beam is described in Table 1. Beam 


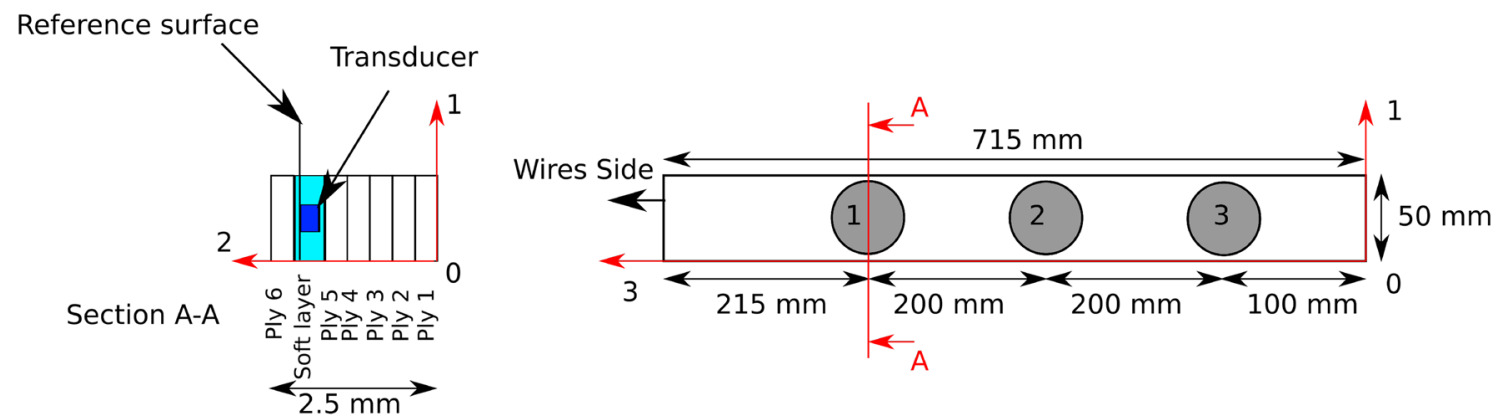

Fig. 1 Technical specifications for the smart composite beams (example corresponding to beam reference f)

Table 1 Location of the piezoelectric transducers for each beam reference (the reference surface is the bottom electrode of the transducers)

\begin{tabular}{lll}
\hline $\begin{array}{l}\text { Beam } \\
\text { refer- } \\
\text { ence }\end{array}$ & $\begin{array}{l}\text { Specific } \\
\text { depth } \\
(\mathrm{mm})\end{array}$ & Location of the transducers \\
\hline $\mathrm{a}$ & 0 & Glued on the top surface of the beam \\
$\mathrm{b}$ & 0.32 & Embedded between the top surface and Ply 1 \\
$\mathrm{c}$ & 0.65 & Embedded between Ply 1 and 2 \\
$\mathrm{~d}$ & 0.98 & Embedded between Ply 2 and 3 \\
$\mathrm{e}$ & 1.32 & Embedded between Ply 3 and 4 \\
$\mathrm{f}$ & 1.98 & Embedded between Ply 5 and 6 \\
\hline
\end{tabular}

(a) is $2 \mathrm{~mm}$ thick because the transducers are glued on the top surface. Consequently, there is no "soft layer". The other beams from (b) to (f) are around $2.5 \mathrm{~mm}$ thick with transducers embedded between plies.

\subsection{Manufacturing process}

The composite manufacturing process is a Vacuum Assisted Resin Transfer Molding (VARTM) [40]. VARTM has been developed as a variant of the traditional Resin Transfer Molding (RTM) process to reduce the cost and design difficulties associated with large metal tools. In VARTM, the upper half of a conventional mold is replaced by a vacuum bag. All the beams are manufactured at the same time in order to guarantee that the beams are nominally identical.

The way of embedding the bulk piezoelectric transducers at the heart of the composite structure is very important to precisely master the position of the transducers inside the structure, in particular along the thickness-axis. The main stages of the manufacturing are described as follows:

1. Preparation of the "soft layer" The piezo ceramics are positioned at the accurate locations on one light glassfiber ply (surface mass of $30 \mathrm{~g} \mathrm{~m}^{-2}$ ). Another light glassfiber ply is positioned on the transducers. This dry device is reinforced with the same polyester resin used

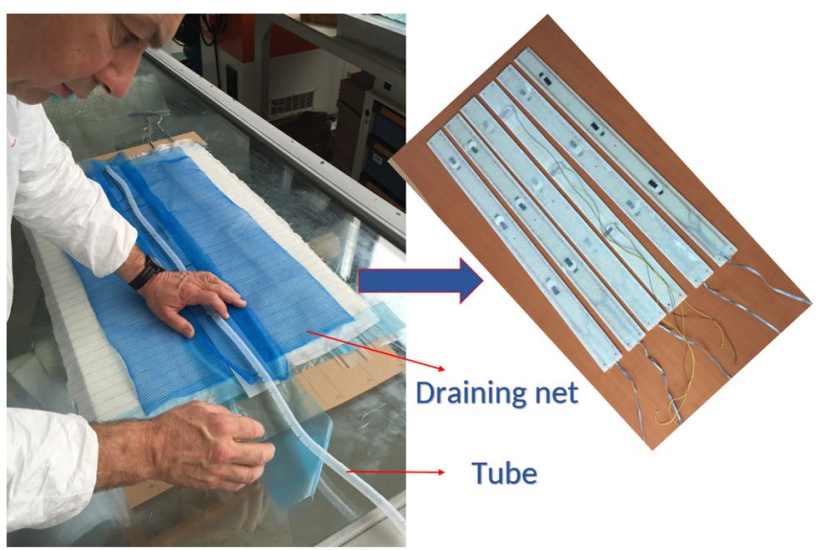

Fig. 2 Manufacturing process

for the whole composite structure in order to guarantee the continuity of the material properties.

2. Laminate preparation Six layers of glass mat are used (surface mass of $300 \mathrm{~g} \mathrm{~m}^{-2}$ ). The "soft layer" is put between two fiber layers. According to the technical requirements, the transducer location is accurately guaranteed by using the "soft layer" [24]. Then the matrix is reinforced by a polyester resin.

3. Manufacturing process After organizing the laminates, a draining net as well as a tube are used to ensure the resin can feed every part of the model, as shown in Fig. 2. The draining net and the feeding tube are put on the top surface of the laminates. A vacuum bag is positioned at the end, covering all the composite structure. A pump is used to achieve full vacuum in order to compact the fibers and the resin. After the curing, a large plate is demolded. This plate is finally machined to obtain 6 beams.

\section{Experimental characterization method}

In this section, the experimental set-up, the characterization method and a data correction method are described. 


\subsection{Measurement apparatus and characterization method}

In Fig. 3, the experimental setup is presented. The test beams are hanged by two wires in order to approximate free boundary conditions. The deflection shapes of the beams are measured with a scanning vibrometer (Polytec, PSV-500-3D). The frequency range is from 1 to $1200 \mathrm{~Hz}$ with a step of $0.3 \mathrm{~Hz}$. The piezo-ceramics are used one after the other as actuators. 105 scan points ( 21 by 5 grid) are measured on each beam. The eigenfrequencies, the modal damping ratio and the vibration amplitudes from the first five natural bending modes of each beam are extracted and analyzed. In order to extract the damped parameters from the measurements, a method of reconstruction of the damped vibration behavior is used $[9,13,19]$ via a modal analysis software package called MODAN [35]. The leastsquare complex frequency domain (LSCF) method is used for modal identification $[33,44]$.

\subsection{Mass and elastic properties}

First of all, it is necessary to identify the mass density and the fibre volume ratio for the composite material manufactured. As the fiber volume ratio is an important input data for the composite models, a ThermoGravimetric Analysis (TGA) is achieved. At the end of this analysis, the weight ratio of the glass fibers is measured at $57.8 \%$. The mass density of the glass fibers is $2600 \pm 3 \% \mathrm{~kg} \cdot \mathrm{m}^{-3}$ and that of the thermosetting plastic is around $1100 \pm 3 \% \mathrm{~kg} \cdot \mathrm{m}^{-3}$. Therefore, the glass fiber volume ratio for the composite material is $37 \%$. The mass density is classically measured at around $1630 \pm 3 \% \mathrm{~kg} \cdot \mathrm{m}^{-3}$. Concerning the "soft layer", the mass density is around $1150 \pm 3 \% \mathrm{~kg} \cdot \mathrm{m}^{-3}$.

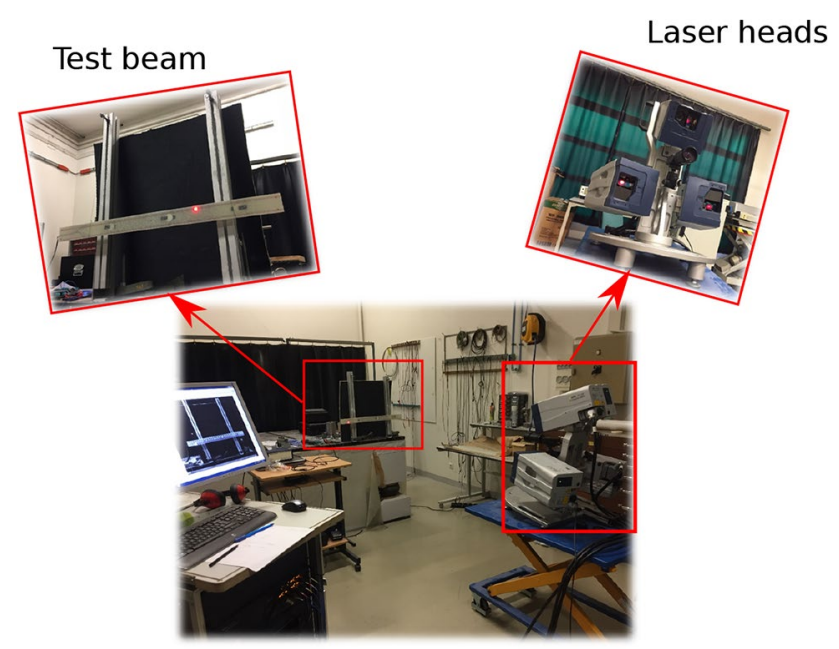

Fig. 3 Experimental setup for identification
From the experimental natural bending modes, the Resonalyser method can be used to extract the composite material parameters $[12,26]$. This method is based on the calibration of a numerical vibration behavior from a representative model with respect to the experimental vibration behavior. A typical frequency response function of the beams studied are depicted in Fig. 4. This calibration needs an optimization process on the material parameters in order to minimize the error between the numerical resonance frequencies and the experimental resonance frequencies. This method is well-adapted for the identification of global elastic properties.

Concerning the constitutive equation, several assumptions can be formulated. On the one hand, due to the characteristics of the laminate manufactured, the homogenized material can be considered transversely isotropic with respect to the out-of-plane axis $[6,7]$. On the other hand, due to the dimensions of the test structures, the assumption of plane stress can be considered. Consequently, the constitutive law is formulated under the reduced form presented in Eq. (1).

$\left\{\begin{array}{c}\epsilon_{1} \\ \epsilon_{2} \\ \gamma_{12}\end{array}\right\}=\left[\begin{array}{ccc}\frac{1}{E} & \frac{-v}{E} & 0 \\ \frac{-v}{E} & \frac{1}{E} & 0 \\ 0 & 0 & \frac{2(1+v)}{E}\end{array}\right]\left\{\begin{array}{c}\sigma_{1} \\ \sigma_{2} \\ \tau_{12}\end{array}\right\}$

where $\epsilon_{i}$ is the longitudinal strain component along the $\mathrm{i}$-axis $(-), \gamma_{i j}$ the shear strain component in the ij-plane $(-), \sigma_{i}$ the longitudinal stress component along the $\mathrm{i}$-axis $\left(\mathrm{N} \mathrm{m}^{-2}\right), \tau_{i j}$ the shear stress component in the ij-plane $\left(\mathrm{N} \mathrm{m}^{-2}\right), E\left(=E_{i}=E_{j}\right)$ the homogenized Young's modulus $\left(\mathrm{N} \mathrm{m}^{-2}\right)$ and $v\left(=v_{i j}\right)$ the homogenized Poisson's ratio.

The Resonalyser method is applied to extract the material parameters. For the composite material, the Young's modulus of the composite material is measured at $14.6 \pm 6.2 \% \mathrm{GPa}$ and the Poisson's ratio $0.24 \pm 2 \%$. For the "soft layer", the Young's modulus is measured at $7 \pm 6.2 \%$ GPa and the Poisson's ratio $0.32 \pm 2 \%$.

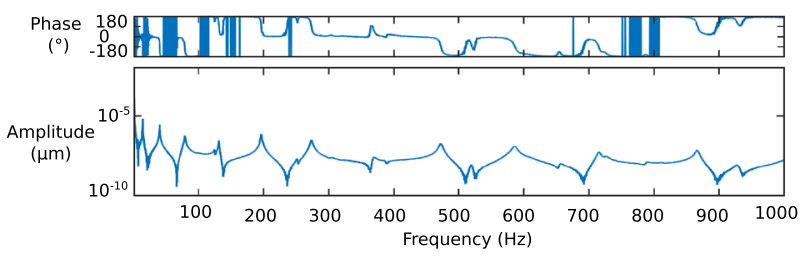

Fig. 4 Typical frequency response function of the beams studied 


\subsection{Data correction of the experimental results}

Due to the geometric uncertainties (length, width and thickness) from the manufacturing process, the geometry of the test beams is not strictly equivalent. In order to preserve the possibility to directly compare the results obtained, it is necessary to correct these deviations by applying a specific computation process. A finite element model is built, which is used as a reference to correct the data from each beam, in order to ensure that all the compared beams have the same length, width and thickness. A $2 \mathrm{D}$ model is built according to a cut-plane along the length axis and the thickness axis. Transducers are not modeled. Indeed, in view of the thicknesses involved and the stiffness ratios, the "soft layer" is the main responsible for the variation of the natural frequencies. The material parameters applied are from the identification done in the last subsection with the Resonalyser method. Second-order quadratic rectangular elements are used for the mesh. A mapped quadrilateral mesh with 15 by 9 rectangle grid (15 along the length and 9 along the thickness with 3 per layer) is used, as depicted in Fig. 5. The blue part is the "soft layer" and the grey part is composite. This mesh is optimized with a convergence analysis as shown in Fig. 6 . The reference for the error computation is the eigenfrequencies obtained for 40 finite elements along the beam axis.

A specific procedure is developed.

1. A first finite element model is built with the real dimensions of the beam studied The eigenfrequencies are computed and stored.

2. The dimensions of this finite element model are modified to obtainthe "ideal" ones $\left(715 \times 50 \times 2.5 \mathrm{~mm}^{3}\right)$ The computation is launched again and new eigenfrequencies are obtained (called "ideal" eigenfrequencies).

3. For each test beam and for each natural mode, a correction value is calculated from the ratio between the "ideal" eigenfrequency and the initial eigenfrequency

4. These correction values are applied to the measured eigenfrequencies for the specific test beam and for each mode.

The results of data correction are shown in Tables 2 and 3: the left side shows the initial measured data for which the

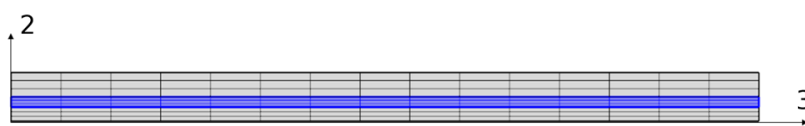

Fig. 5 Mesh used with a scale factor along the thickness axis (the blue area is the "soft layer")

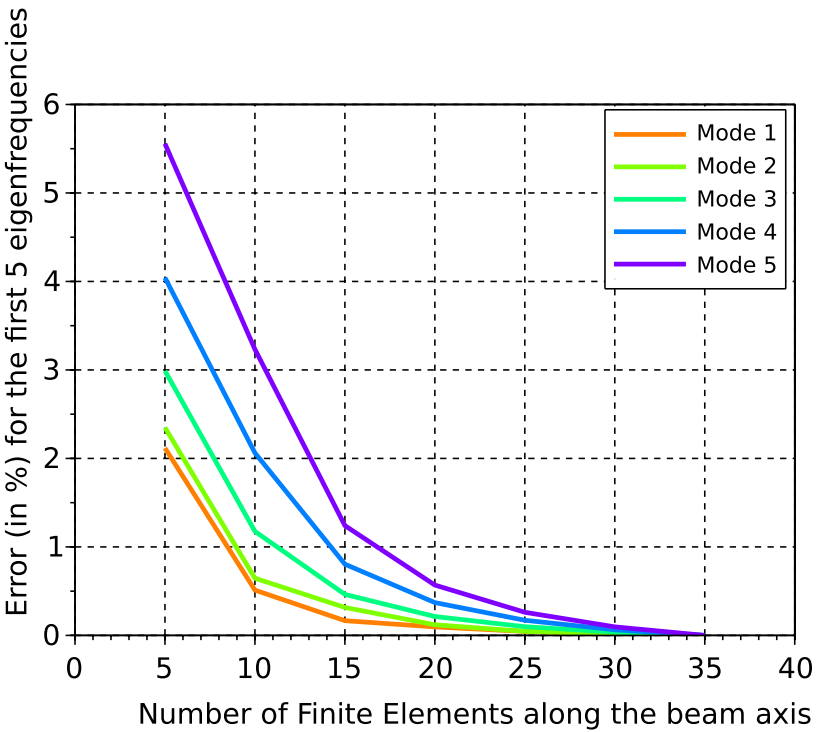

Fig. 6 Convergence curve for the first five eigenfrequencies

length and thickness of each beam are not accurately equal; the right part is the results after correction.

In this analysis, only the first five bending modes are considered. The corresponding shapes for Beam (c) are illustrated in Fig. 7. For the other beams, the effect of the location of the "soft layer"' through the thickness have a very low impact on the global mode shapes. Furthermore, the thickness ratio between the piezoelectric transducers and the beam is very low. Consequently, the presence of the transducers does not modify the mechanical behavior of the beam [25].

\subsection{Repeatability analysis}

In order to confirm the results, an additional rush of beams have been processed, and all the experiments have been repeated. The Repeatability Standard Deviation (RSD) from the results is computed and given for all the investigations. Of course, the changes in the data observed between the reference beams must be much higher than this value in order to be relevant and representative. Moreover, 5 more samples of Beam (c) are manufactured with a position variation with respect to the feeding tube in order to guarantee that the Vacuum Assisted Resin Transfer Molding (VARTM) has no impact on the beams parameters. These 5 beams are tested following the same experimental procedure, in order to validate the repeatability and accuracy of the manufacturing process. No significant variation is obtained. 
Table 2 Initial (I) and corrected (C) data for each beam reference from a to $c$

\begin{tabular}{|c|c|c|c|c|c|c|}
\hline \multirow{2}{*}{$\begin{array}{l}\text { Beam reference } \\
\text { Initial (I) or corrected } \\
\text { (C) data }\end{array}$} & \multicolumn{2}{|l|}{ a } & \multicolumn{2}{|l|}{$\mathrm{b}$} & \multicolumn{2}{|l|}{ c } \\
\hline & I & C & 1 & $C$ & 1 & C \\
\hline Length (mm) & 713 & 715 & 715 & 715 & 713 & 715 \\
\hline Width (mm) & 50 & 50 & 50 & 50 & 50 & 50 \\
\hline Thickness (mm) & 2.03 & 2.5 & 2.48 & 2.5 & 2.4 & 2.5 \\
\hline $\mathrm{F} 1(\mathrm{~Hz})$ & 11.37 & 13.93 & 12.57 & 12.68 & 13.27 & 13.70 \\
\hline F2 (Hz) & 31.59 & 38.68 & 34.93 & 35.23 & 36.85 & 38.05 \\
\hline F3 (Hz) & 62.66 & 76.70 & 69.28 & 69.87 & 73.08 & 75.46 \\
\hline $\mathrm{F} 4(\mathrm{~Hz})$ & 105.23 & 128.76 & 116.32 & 117.31 & 122.69 & 126.67 \\
\hline F5 (Hz) & 160.30 & 196.06 & 177.15 & 178.65 & 186.82 & 192.87 \\
\hline
\end{tabular}

Table 3 Initial (I) and corrected (C) data for each beam reference from $d$ to $f$

\begin{tabular}{|c|c|c|c|c|c|c|}
\hline \multirow{2}{*}{$\begin{array}{l}\text { Beam reference } \\
\text { Initial (I) or corrected } \\
\text { (C) data }\end{array}$} & \multicolumn{2}{|l|}{$d$} & \multicolumn{2}{|l|}{ e } & \multicolumn{2}{|l|}{$\mathrm{f}$} \\
\hline & 1 & $\mathrm{C}$ & 1 & $\mathrm{C}$ & I & $\mathrm{C}$ \\
\hline Length (mm) & 712 & 715 & 714 & 715 & 715 & 715 \\
\hline Width (mm) & 50 & 50 & 50 & 50 & 50 & 50 \\
\hline Thickness (mm) & 2.39 & 2.5 & 2.53 & 2.5 & 2.51 & 2.5 \\
\hline $\mathrm{F} 1(\mathrm{~Hz})$ & 13.82 & 14.28 & 14.67 & 14.46 & 13.78 & 13.70 \\
\hline $\mathrm{F} 2(\mathrm{~Hz})$ & 38.39 & 39.65 & 40.74 & 40.16 & 38.26 & 38.05 \\
\hline F3 $(\mathrm{Hz})$ & 76.12 & 78.61 & 80.77 & 79.63 & 75.87 & 75.46 \\
\hline $\mathrm{F} 4(\mathrm{~Hz})$ & 127.76 & 131.94 & 135.55 & 133.64 & 127.36 & 126.67 \\
\hline F5 (Hz) & 194.51 & 200.85 & 206.33 & 203.43 & 193.91 & 192.87 \\
\hline
\end{tabular}

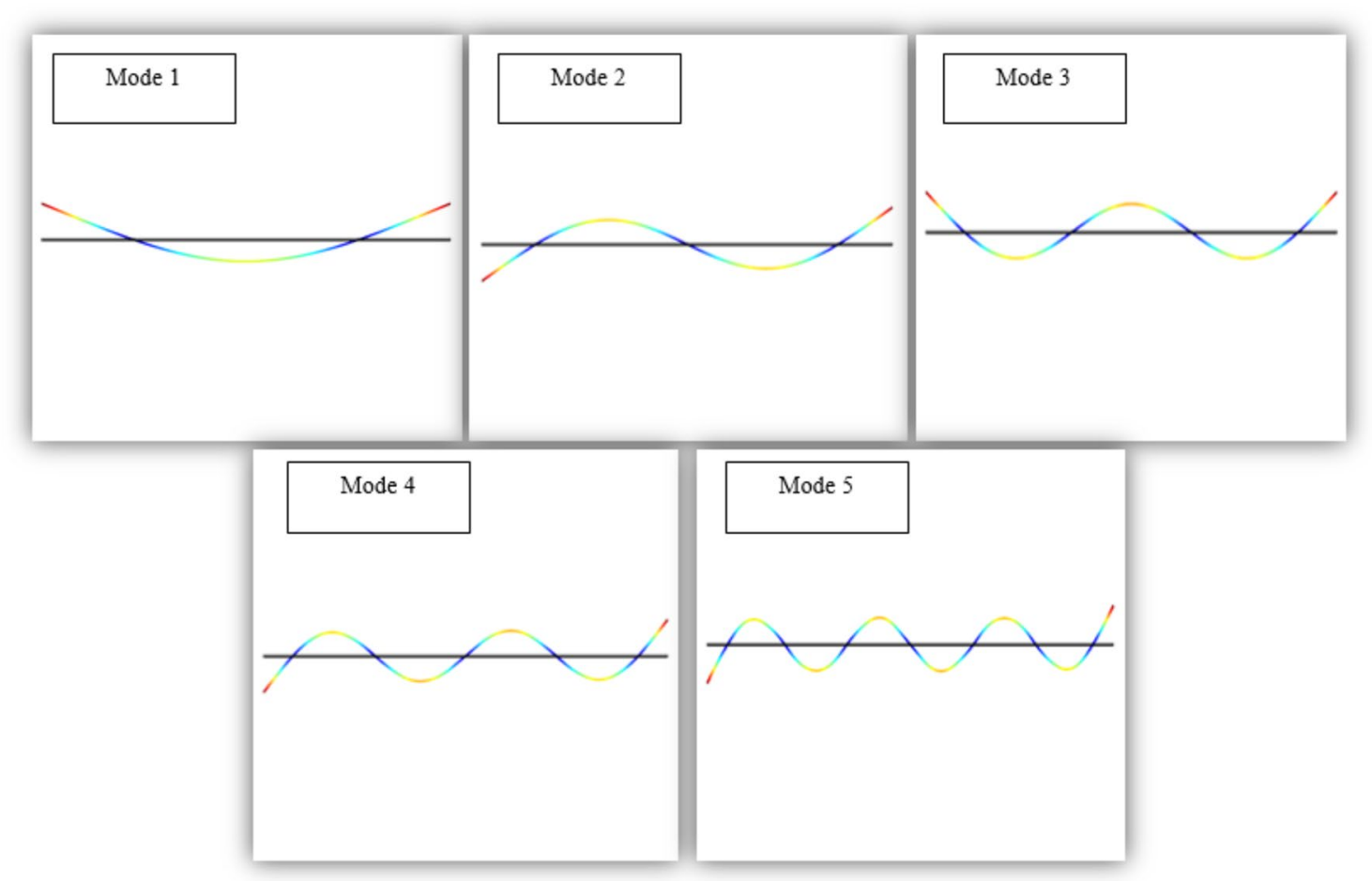

Fig. 7 First five natural elastic bending mode shapes of beam (c) from the numerical modeling 
Table 4 Corrected experimental eigenfrequencies for each beam reference and maximal standard deviation for each natural vibration frequency and all beam references

\begin{tabular}{lrrrrrrr}
\hline Beam reference & a & \multicolumn{1}{l}{$\mathrm{b}$} & $\mathrm{c}$ & $\mathrm{d}$ & $\mathrm{e}$ & $\mathrm{f}$ & $\mathrm{RSD}(\%)$ \\
\hline F1 (Hz) & 13.59 & 13.77 & 14.71 & 15.13 & 15.60 & 14.63 & 0.12 \\
F2 (Hz) & 37.36 & 38.12 & 40.53 & 42.12 & 42.52 & 39.99 & 0.05 \\
F3 (Hz) & 73.24 & 74.66 & 79.29 & 81.49 & 82.46 & 78.90 & 0.08 \\
F4 (Hz) & 121.72 & 125.07 & 128.33 & 136.15 & 137.57 & 131.08 & 0.04 \\
F5 (Hz) & 180.59 & 186.70 & 200.27 & 202.57 & 207.08 & 196.93 & 0.08 \\
\hline
\end{tabular}

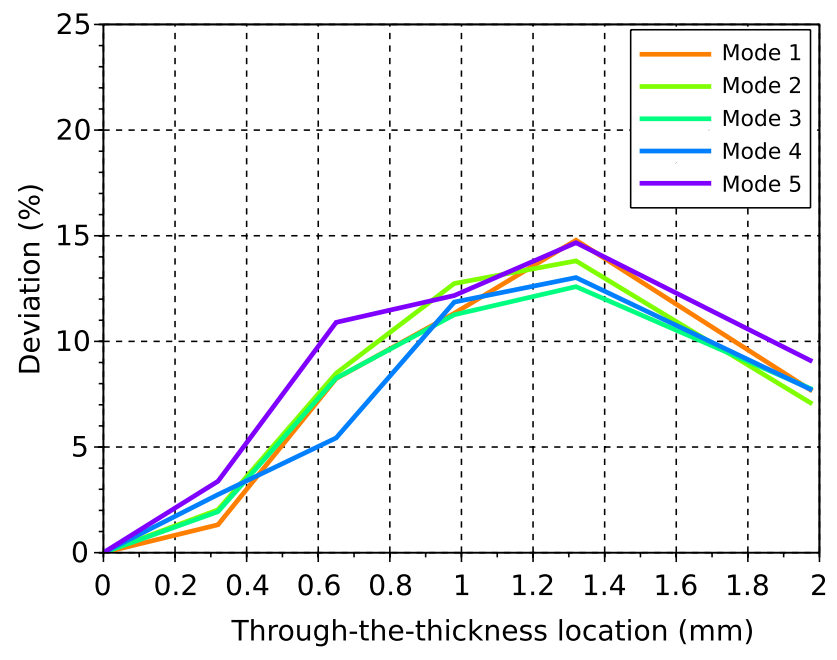

Fig. 8 Relative evolution of the eigenfrequencies versus location of the "soft layer" for the first 5 bending modes

\section{Results and discussion}

In order to experimentally investigate the influence of the "soft layer" location along the thickness-axis on the performance of the smart composite structures, several design parameters have be investigated such as the eigenfrequencies, the modal effective electromechanical coupling coefficient, the modal damping ratios, the vibration amplitude and the Lamb waves propagation.

\subsection{Natural bending frequencies}

In Table 4, the first five experimental eigenfrequencies are given after data correction. The given RSD corresponding to the maximum variability for each vibration mode by considering all the beam references is also provided.

Figure 8 presents the relative deviation of the eigenfrequencies with respect to the eigenfrequencies obtained for Beam (a) in function of the location of the "soft layer". For all beams, the tendency for each eigenfrequency is similar. The maximum value of the eigenfrequency is obtained when the "soft layer" is embedded in
Table 5 Eigenfrequencies when Piezo 1 is short-circuited (SC) or open-circuited (OC) for beam $d$ and $f$

\begin{tabular}{|c|c|c|c|c|c|}
\hline \multirow{2}{*}{$\begin{array}{l}\text { Beam reference } \\
\text { Short-circuit (SC) or } \\
\text { open-circuit (OC) }\end{array}$} & \multicolumn{2}{|l|}{$d$} & \multicolumn{2}{|l|}{$f$} & \multirow[t]{2}{*}{ RSD $(\%$} \\
\hline & OC & SC & OC & SC & \\
\hline $\mathrm{F} 1(\mathrm{~Hz})$ & 14.64 & 14.64 & 14.02 & 14.21 & 0.12 \\
\hline $\mathrm{F} 2(\mathrm{~Hz})$ & 41.18 & 41.17 & 39.49 & 39.39 & 0.05 \\
\hline F3 $(\mathrm{Hz})$ & 81.47 & 81.47 & 78.08 & 78.05 & 0.08 \\
\hline $\mathrm{F} 4(\mathrm{~Hz})$ & 135.50 & 135.54 & 130.09 & 130.06 & 0.04 \\
\hline $\mathrm{F} 5(\mathrm{~Hz})$ & 202.71 & 202.79 & 196.04 & 195.98 & 0.08 \\
\hline
\end{tabular}

the middle of the composite structure. The highest variation is above $10 \%$ with regards to the standard setup when the transducers are glued on the top surface of the beam. This fact is explained by the modification of the second moment of area due to the through-thethickness location of the "soft layer".

Furthermore, even though only bending modes are considered in this paper, it should be mentioned that Beam (d) ("soft layer" at $0.98 \mathrm{~mm}$ depth) and Beam (e) ("soft layer" at $1.32 \mathrm{~mm}$ depth) exhibit torsion modes in the frequency bandwidth, respectively at $124.3 \mathrm{~Hz}$ and $132.4 \mathrm{~Hz}$. When the transducers are located in the middle plane of the beam, their effect is very limited to actuate the bending modes of the beam. Consequently, with the unavoidable position uncertainty along the beam-axis, the piezoelectric transducers can also actuate torsion modes.

\subsection{Modal effective electromechanical coupling coefficient}

Probably the most interesting method to evaluate the effective electromechanical coupling coefficients (EMCC) is based on the effective energy conversion from mechanical to electrical and vice-versa for the whole system [42]. For this part, the eigenfrequencies state is measured when Piezo 1 is short-circuited (SC) or open-circuited (OC). Beam $\mathrm{d}$ is chosen as an example, Piezo 1 is used as an actuator, the results are shown in Table 5, as well as the deviation of the repetition experiments.

When the electric boundary conditions of Piezo 1 is modified, the eigenfrequencies are slightly changed. But 
Table 6 Maximal amplitudes for each beam reference and for each natural mode

\begin{tabular}{lcclllll}
\hline Beam reference & $\mathrm{a}$ & $\mathrm{b}$ & $\mathrm{c}$ & $\mathrm{d}$ & $\mathrm{e}$ & $\mathrm{f}$ & $\mathrm{RSD}(\%)$ \\
\hline Mode 1 amplitude $(\mu \mathrm{m})$ & 32.7 & 14.6 & 6.5 & 7.06 & 0.49 & 3.27 & 3.42 \\
Mode 2 amplitude $(\mu \mathrm{m})$ & 11.9 & 6.03 & 4.83 & 2.21 & 0.16 & 5.46 & 3.44 \\
Mode 3 amplitude $(\mu \mathrm{m})$ & 4.37 & 5.47 & 2.76 & 0.7 & 0.08 & 1.24 & 3.84 \\
Mode 4 amplitude $(\mu \mathrm{m})$ & 2.69 & 1.81 & 0.91 & 0.95 & 0.09 & 1.61 & 0.91 \\
Mode 5 amplitude $(\mu \mathrm{m})$ & 1.95 & 1.36 & 0.67 & 0.5 & 0.04 & 1.11 & 0.43 \\
\hline
\end{tabular}

Table 7 Modal damping ratios for each beam reference

\begin{tabular}{lllllllr}
\hline Beam reference & $\mathrm{a}$ & $\mathrm{b}$ & $\mathrm{c}$ & $\mathrm{d}$ & $\mathrm{e}$ & $\mathrm{f}$ & $\mathrm{RSD}(\%)$ \\
\hline Mode 1 damping ratio (\%) & 0.58 & 0.47 & 0.55 & 0.51 & 0.53 & 0.32 & 4.93 \\
Mode 2 damping ratio (\%) & 0.51 & 0.51 & 0.5 & 0.65 & 0.41 & 0.5 & 2.02 \\
Mode 3 damping ratio (\%) & 0.53 & 0.38 & 0.41 & 0.84 & 0.42 & 0.88 & 4.39 \\
Mode 4 damping ratio (\%) & 0.25 & 0.44 & 0.44 & 0.43 & 0.39 & 0.48 & $\approx 0.00$ \\
Mode 5 damping ratio (\%) & 0.47 & 0.61 & 1.2 & 0.4 & 0.37 & 0.44 & 1.21 \\
\hline
\end{tabular}

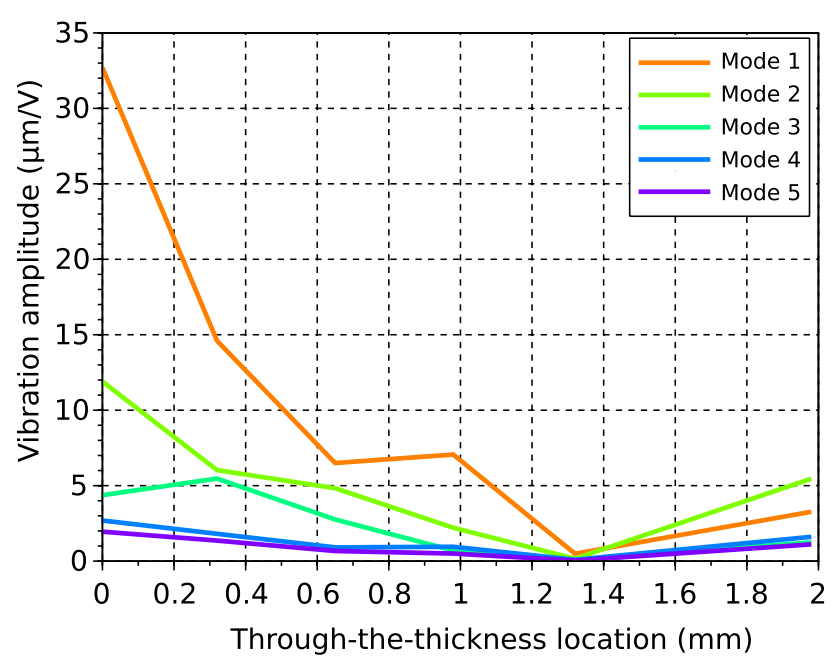

Fig. 9 Modal amplitude versus location of the "soft layer" for the first 5 bending modes

by comparing with the Repeatability Standard Deviation, the eigenfrequency deviation is not significant enough. So it is not possible to evaluate the EMCC in this situation. In conclusion, the smart composite structures are considered a weakly coupled.

\subsection{Modal damping ratio and vibration amplitude}

In this section, the maximum amplitudes and the damping ratios for each natural mode and for each beam reference are given respectively in Table 6 and in Table 7.

Concerning the modal amplitudes, the tendency is equivalent for all the eigenfrequencies as shown in Fig. 9. The minimal modal amplitude is obtained when the "soft layer" is at the middle of the beam and the maximal one when the "soft layer" is located at the top surface of the beams. The result is mechanically obvious if the "soft layer" is not neglicted with respect to the composite material.

The "soft layer" is mainly made of resin. So, one could expect that this layer increases the damping ratio of the beam. But, it seems not to be the case. In Table 7, if Beam (a) is compared to the other references, there is no effect or particular tendency observed. Moreover, the position of the "soft layer" has no obvious impact of the damping ratio.

\subsection{Lamb waves propagation}

Time-of-flight Method is a material characterization method, which exploits the ultrasonic wave propagation properties [43]. A wave train, typically a sinusoidal burst, is generated with an actuator and a sensor measures the time of flight of this wave train, as depicted in Fig. 10. As the measured phase velocity of $S_{0}$ symmetrical lamb wave train, called $c_{p h}^{S_{0}}\left(\mathrm{~m} \mathrm{~s}^{-1}\right)$, is related to the mechanical properties, the Young's modulus, E, of the material measured can be calculated by

$c_{p h}^{S_{0}}=\sqrt{\frac{E}{\rho \cdot\left(1-v^{2}\right)}}$

where $\rho$ is the mass density $\left(\mathrm{Kg} \mathrm{m}^{-3}\right)$.

To generate and capture the wave trains, the piezoelectric transducers embedded in the composite are used. The advantage of this method compared with Resonalyser Method is that T-o-F Method is easier and faster to extract the material properties along the propagation axis. But, it is necessary to evaluate the Poisson's ratio. The Poisson's ratio of the glass fiber is around 0.23 and that of the 


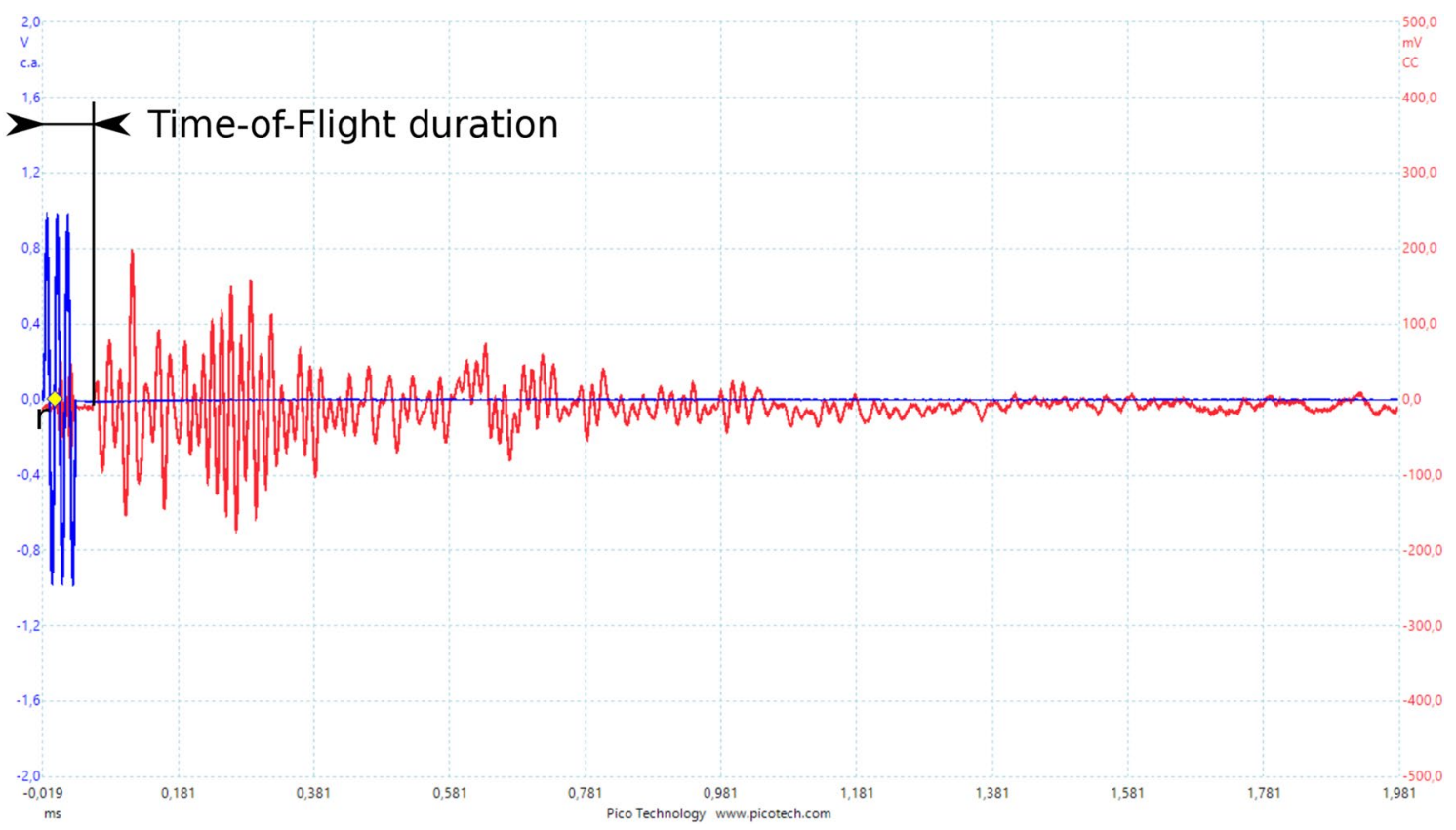

Fig. 10 Definition of the time-of-flight between the actuator signal (blue line) and the sensor signal (red line)

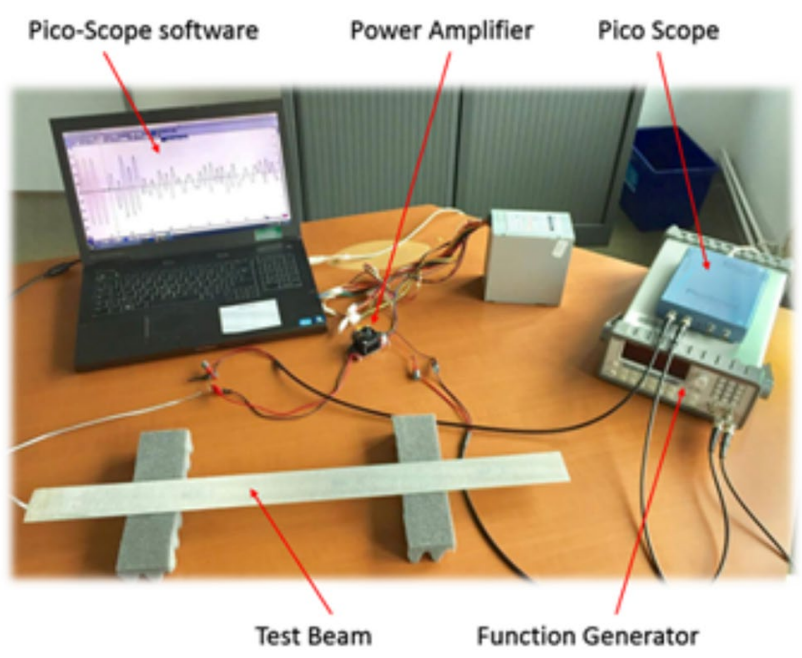

Fig. 11 Experimental setup for time-of-flight method

thermosetting plastic around 0.37 , so the Poisson's ratio can be evaluated at 0.32 with the classical rule of mixtures [15].

Figure 11 shows the experimental setup to measure the wave trains. A function generator (Keithley, 3390) is used to generate excitation signals via a miniature power amplifier (PiezoDrive, PDM200B). The signals are then captured (PicoScope, 4424), and analyzed by PicoScope software. One of the piezo electric transducers (No.1) is used as actuator. After a set of signal optimization tests, the aim of which being to optimize the response signals, a short number of sinusoidal bursts ( 1 to 3 cycles) are chosen as excitation signals. Another piezo electric transducer (No.3) is used as sensor, the time-of-flight from the actuator to the sensor is then recorded for each beam.

The goal of this investigation is to evaluate the influence of the through-the-thickness location of the "soft layer" with respect to the values of the time of flight. Table 8 gives the experimental results. There is no relevant tendency. The "soft layer" and its location seem to have a very limited influence on these results. Indeed, the Lamb waves propagate along the beam-axis under two forms of motion: a symmetrical motion about the midplan of the beam and a antisymmetric motion about the midplane. In this investigation, only the symmetric mode (the speediest one) is used. This mode is less sensitive to the variation of the second moment of area compared with the bending modes. From Eq. (2), the Young's modulus of the composite material is calculated at $14.5 \pm 1.1 \% \mathrm{GPa}$. This value is in good agreement
Table 8 Time-of-flight values for each beam reference

\begin{tabular}{llllllll}
\hline Beam reference & a & b & c & d & e & f & RSD (\%) \\
\hline Time-of-flight $(\mu \mathrm{s})$ & 126 & 125 & 126 & 126 & 128 & 127 & 0.08 \\
\hline
\end{tabular}


with the Young's modulus obtained by the resonalyzer method.

\section{Conclusion}

The work is focused on smart composite structures and, in particular, composite structures activated by a "soft layer" containing transducers. The test structures are beams made of Glass Fibers Reinforced Polymer (GFRP) laminates. The influence of the through-the-thickness position of the "soft layer" on the structural performance of the beams was experimentally investigated. The eigenfrequencies, the modal amplitudes, the damping ratios and the Time of flight of the Lamb waves are analyzed. Five different beam setups were tested and compared to the standard setup when the transducers are glued on the top surface of the beam. Repeatability tests to calculate the Repeatability Standard Deviation (RSD) for each result were done as well as repeatability tests in order to evaluate the stability of the manufacturing process. A numerical method based on a finite element model has been developed to take into account the geometry variation.

The results demonstrated that the "soft layer" can not be neglicted to model the behavior of the final product. In particular, the through-the-thickness position has an influence of the eigenfrequencies and the modal amplitudes. The maximal frequencies is obtained when the "soft layer" is located at the middle of the beam. The maximal modal amplitude is obtained when the "soft layer" is at the top surface of the beam. However, the "soft layer" does not increase the overall damping ratio of the final structures and the through-the-thickness position of the "soft layer" has no influence on the damping ratios. The Lamb wave propagation inside the composite material is not impacted the "soft layer". This data is important in particular to design Stuctural Health Monitoring (SHM) strategies based on Lamb waves. The results obtained can constitute an experimental benchmark data that will be useful for validation of computational codes or model developments.

Acknowledgements This project has been performed in cooperation with the EUR EIPHI program (Contract ANR 17-EURE-0002). This work was partly supported by a financial support from the UTBM, France and is part of the collaborative project named SyRaCuSE (SmaRt Composite StructurE). The authors are grateful to Mr Romain Viala from the FEMTO-ST Institute for his help and his fruitful assistance on modal analysis. The first author thanks the China Scholarship Council for financial support gratefully (Contract No. 201504490012).

\section{Compliance with ethical standards}

Conflict of interest The authors declare that they have no conflict of interest.

\section{References}

1. Agarwal BD, Broutman LJ, Chandrashekhara K (2017) Analysis and performance of fiber composites. Wiley, New York

2. Akhras G (2000) Smart materials and smart systems for the future. Can Mil J 1(3):25-31

3. Calvary G, Delot T, Sedes F, Tigli JY (2013) Computer science and ambient intelligence. Wiley, New York

4. Campbell FC (2010) Structural composite materials. ASM International, Materials Park

5. Cheah LW (2010) Cars on a diet: the material and energy impacts of passenger vehicle weight reduction in the us. PhD thesis, Massachusetts Institute of Technology

6. Chen X, Lachat R, Salmon S, Ouisse M, Meyer Y (2017a) Complex composite structures with integrated piezoelectric transducers. Int J Comput Methods Exp Meas 5(2):125-134

7. Chen X, Meyer Y, Lachat R, Ouisse M (2017b) Laminates with integrated piezoelectric transducers:influence of the transducers location along the thickness-axis on the structural performance. In: International center for numerical methods in engineering (CIMNE), VIII ECCOMAS thematic conference on smart structures and materials (SMART 2017), pp 594-605

8. Chow W, Graves M (1992) Stress analysis of a rectangular implant in laminated composites using 2-d and 3-d finite elements. In: 33 rd structures, structural dynamics and materials conference, $\mathrm{p} 2477$

9. Chu M, Golub G, Golub GH (2005) Inverse eigenvalue problems: theory, algorithms, and applications, vol 13. Oxford University Press, oxford

10. Collet M, Ouisse M, Tateo F (2014) Adaptive metacomposites for vibroacoustic control applications. IEEE Sens J 14(7):2145-2152

11. Crawley E, Luis DE (1987) Use of piezoelectric actuators as elements of intelligent structures. AIAA J 25(10):1373-1385

12. De Visscher J, Sol H, De Wilde W, Vantomme J (1997) Identification of the damping properties of orthotropic composite materials using a mixed numerical experimental method. Appl Compos Mater 4(1):13-33

13. Foltete E, Gladwell G, Lallement G (2001) On the reconstruction of a damped vibrating system from two complex spectra, part 2: experiment. J Sound Vib 240(2):219-240

14. Gandhi MV, Thompson B (1992) Smart materials and structures. Springer Science \& Business Media, Berlin

15. Gay D (2014) Composite materials: design and applications. CRC Press, Boca Raton

16. George HS (1999) Laminate analysis. Laminar composites, Boston

17. Ghezzo F, Huang Y, Nemat-Nasser S (2009) Onset of resin micro-cracks in unidirectional glass fiber laminates with integrated shm sensors: experimental results. Struct Health Monit 8(6):477-491

18. Giurgiutiu V, Zagrai A, Jing Bao J (2002) Piezoelectric wafer embedded active sensors for aging aircraft structural health monitoring. Struct Health Monit 1(1):41-61

19. Gladwell G (2001) On the reconstruction of a damped vibrating system from two complex spectra, part 1: theory. J Sound Vib 240(2):203-217

20. Hansen J, Vizzini A, Hansen J, Vizzini A (1997) Fatigue response of a host structure with interlaced embedded devices. In: 38th structures, structural dynamics, and materials conference

21. Hufenbach W, Böhm R, Thieme M, Tyczynski T (2011) Damage monitoring in pressure vessels and pipelines based on wireless sensor networks. Proced Eng 10:340-345

22. Hull D, Clyne T (1996) An introduction to composite materials. Cambridge University Press, Cambridge 
23. Kapuria S (2001) An efficient coupled theory for multilayered beams with embedded piezoelectric sensory and active layers. Int J Solids Struct 38(50-51):9179-9199

24. Lachat $R$, Meyer $Y$ (2017) Method for producing a part made of a composite material, incorporating components and marking means. WO Patent WO/2017/013346

25. Lachat R CW Borza D (2004) Manufacture of smart composite panels for the industry and verification of teir structural properties with holographic methods. In: ICEM12-12th international conference on experimental mechanics, Bari, Italy, p 8

26. Lauwagie T (2005) Vibration-based methods for the identification of the elastic properties of layered materials (trillingsgebaseerde methodes voor de identificatie van de elastische eigenschappen van gelaagde materialen). PhD thesis, Katholieke Universiteit Leuven

27. Lin M, Chang FK (2002) The manufacture of composite structures with a built-in network of piezoceramics. Compos Sci Technol 62(7-8):919-939

28. Meyer Y, Lachat R (2015) Vibration characterization procedure of piezoelectric ceramic parameters-application to low-cost thin disks made of piezoceramics. In: MATEC web of conferences, EDP Sciences, vol 20, p 01003

29. Meyer Y, Lachat $R$ (2016) Smart composite structures: benefits, technical issues and potential applications. JEC Compos Mag 105

30. Meyer $Y$, Lachat $R$, Chen $X$, Salmon S, Ouisse M (2017) Smart composite structure (project syracuse) towards mastering challenges concerning the product lifecycle. In: 20th international conference on composite structures (ICCS20), Paris

31. Paradies R (1997) Statische verformungskontrolle hochgenauer faserverbundreflektorschalen mit hilfe aktiver elemente. $\mathrm{PhD}$ thesis, ETH Zurrich, Switzerland

32. Paradies $R$, Ruge $M(2000)$ In situ fabrication of active fibre reinforced structures with integrated piezoelectric actuators. Smart Mater Struct 9(2):220

33. Peeters B, Van der Auweraer H, Guillaume P, Leuridan J (2004) The polymax frequency-domain method: a new standard for modal parameter estimation? Shock Vib 11(3,4):395-409

34. Phung-Van $P$, De Lorenzis $L$, Thai $\mathrm{CH}$, Abdel-Wahab $M$, NguyenXuan H (2015) Analysis of laminated composite plates integrated with piezoelectric sensors and actuators using higherorder shear deformation theory and isogeometric finite elements. Comput Mater Sci 96:495-505
35. Piranda J (2001) Analyse modale expérimentale. Techniques de l'ingénieur Bruit et vibrations 6180:1776-0143

36. Qing XP, Beard SJ, Kumar A, Ooi TK, Chang FK (2007) Built-in sensor network for structural health monitoring of composite structure. J Intell Mater Syst Struct 18(1):39-49

37. Sala G, Olivier M, Bettini P, Sciacovelli D (2004) Embedded piezoelectric sensors and actuators for control of active composite structures. Mechanical and Thermal Engineering Department, Carlo Gavazzi Space ESTEC, European Space Agency

38. Saravanos DA, Heyliger PR (1995) Coupled layerwise analysis of composite beams with embedded piezoelectric sensors and actuators. J Intell Mater Syst Struct 6(3):350-363

39. Saravanos DA, Heyliger PR, Hopkins DA (1997) Layerwise mechanics and finite element for the dynamic analysis of piezoelectric composite plates. Int J Solids Struct 34(3):359-378

40. Song $X$ (2003) Vacuum assisted resin transfer molding (vartm): model development and verification. PhD thesis, Virginia Tech

41. Taminger K (2012) Technical challenges to reducing subsonic transport weight. In: AIAA aerospace sciences meeting

42. Trindade MA, Benjeddou A (2009) Effective electromechanical coupling coefficients of piezoelectric adaptive structures: critical evaluation and optimization. Mech Adv Mater Struct 16(3):210-223

43. Tucker B (2001) Ultrasonic plate waves in wood-based composite panels. PhD thesis, Washington State University, USA

44. Van Der Auweraer $H$, Guillaume $P$, Verboven $P$, Vanlanduit $S$ (2001) Application of a fast-stabilizing frequency domain parameter estimation method. J Dyn Syst Meas Control 123(4):651-658

45. Witten E, Kraus T, Kühnel M (2016) Composites market report 2016. Federation of Reinforced Plastics, Germany

46. Wlezien R, Horner G, McGowan A, Padula S, Scott M, Silcox R, Simpson J (1998) The aircraft morphing program. In: 39th AIAA/ ASME/ASCE/AHS/asc structures, structural dynamics, and materials conference and exhibit, p 1927

Publisher's Note Springer Nature remains neutral with regard to jurisdictional claims in published maps and institutional affiliations. 